\title{
Cloning and disruption of the UeArginase in Ustilago esculenta: evidence for a role of arginine in its dimorphic transition
}

\author{
Yafen Zhang ${ }^{1 \dagger}$, Min $\mathrm{Wu}^{1+}$, Qianwen $\mathrm{Ge}^{1}$, Mengfei Yang ${ }^{2}$, Wenqiang Xia ${ }^{1}$, Haifeng Cui ${ }^{1}$, Xiaoping Yu', \\ Shangfa Zhang ${ }^{2}$ and Zihong Ye $e^{1 *}$ (i)
}

\begin{abstract}
Background: Ustilago esculenta, a typical dimorphic fungus could infect Zizania latifolia and induce host stem swollen to form an edible vegetable called Jiaobai in China. The strains differentiation especially in the mating ability and pathogenicity is closely related to different phenotypes of Jiaobai formed in the fields. Dimorphic switching, a tightly regulated processes, is essential for the pathogenetic development of dimorphic fungi. In responses to environment cues, dimorphic switching can be activated through two conserved cell signaling pathways-PKA and MAPK pathways. Previous study indicated that exogenous arginine could induce hyphal formation in several dimorphic fungi through hydrolysis by arginase, but inhibit the dimorphic transition of $U$. esculenta. We conducted this study to reveal the function of arginine on dimorphic transition of $U$. esculenta.
\end{abstract}

Results: In this study, we found that arginine, but not its anabolites, could slow down the dimorphic transition of $U$. esculenta proportionally to the concentration of arginine. Besides, UeArginase, predicated coding arginase in $U$. esculenta was cloned and characterized. UeArginase mutants could actually increase the content of endogenous arginine, and slow down the dimorphic transition on either nutritious rich or poor medium. Either adding exogenous arginine or UeArginase deletion lead to down regulated expressions of UePkaC, UePrf1, mfa1.2, mfa2.1, pra1 and pra2, along with an increased content of arginine during mating process.

Conclusion: Results of this study indicated a direct role of arginine itself on the inhibition of dimorphic transition of $U$. esculenta, independent of its hydrolysis by UeArginase.

Keywords: Ustilago esculenta, UeArginase, Arginine metabolism, Dimorphic transition

\section{Background}

Dimorphic switching, known as a tightly regulated processes that switching between hyphal growth and yeastlike state, is essential for the pathogenesis of both animal and plant pathogenic dimorphic fungi [1]. There are many environment cues have been described to be important inducers in the dimorphic switching, such as carbon or nitrogen sources $[2,3]$, temperature [4], $\mathrm{pH}$ [5], growth atmosphere [6], or host signals [5]. However, the cell signaling pathway networks in dimorphic fungi

\footnotetext{
* Correspondence: 13656686088@163.com; zhye@cjlu.edu.cn

'Yafen Zhang and Min Wu contributed equally to this work.

'Zhejiang Provincial Key Laboratory of Biometrology and Inspection \& Quarantine, College of Life Sciences, China Jiliang University, Hangzhou 310018, Zhejiang, China

Full list of author information is available at the end of the article
}

are conserved [5]. The cAMP-dependent protein kinase A (PKA) pathway and the mitogen-activated protein kinase (MAPK) pathway have been proved at the heart of this network, responding to the environment cues to regulate fungal dimorphism [1]. Most fungi exhibit dimorphism in response to distinct carbon or nitrogen sources [3], e. g., in Mucor species, yeast growth is preferred when a fermentable hexose is available [7]. Studies showed that arginine, as a nitrogen source, could induce hyphal formation in several dimorphic fungi $[8,9]$, e. g., $10 \mathrm{mM}$ exogenous arginine could induce the dimorphic switching from yeast to mycelia in Ceratocystis ulmi to cause elm disease [8]; for Candida albicans, an increase in arginine through exogenous addition or endogenous synthesis, could induce germ tube formation to escape

(c) The Author(s). 2019 Open Access This article is distributed under the terms of the Creative Commons Attribution 4.0 International License (http://creativecommons.org/licenses/by/4.0/), which permits unrestricted use, distribution, and 
from macrophage in a density-dependent manner [9]. Further researches have proved that the metabolites of arginine activate adenylyl cyclase to synthesize cAMP, which in turn activates PKA pathway to trigger the morphogenetic switch from yeast to hyphae in C. albicans [9].

In the typical smut fungus $U$. maydis, dimorphism is a particular irreversible growth form that switching from a haploid, unicellular phase to a dikaryotic filamentous stage [1]. It implies a critical role of mating (including cell fusion and hyphal growth in the life cycle) in pathogenetic development [10]. The $a$ genes and Prf1 gene are responsible for mating [11-13], which is regulated by some MAPK pathway members such as Kpp2 and Kpp6 [14], and the cAMP-PKA pathway, in which Adr1 and Ubc1 are involved [15]. Acidic pH [5], pheromone [16], starvation of nitrogen [17], fatty acids as carbon source [18] and plant signals such as hydrophobicity [19] have been discovered to influence the mating process in $U$. maydis. However, there is no report of arginine involved in dimorphism of $U$. maydis and other smut fungi.

Ustilago esculenta, resembling an endophytic smut fungus in a perennial root herb plant Zizania latifolia $[20,21]$, would inhibit host flowering and induce host stem swollen to form a flavored vegetable in Southeast Asian [22, 23]. Similarly to $U$. maydis, $U$. esculenta undergoes a dimorphism process from a saprophytic yeast-like haploid stage to a pathogenic heterokaryotic mycelial stage [24, 25]. Evidences showed that dimorphism transition of $U$. esculenta was started after pheromone-receptor recognition and followed by conjugation tubes formation and cells fusion [26]. This process was determined by compatible $a$ genes and regulated by UePrf1, which can interact with UeKpp2 and UeKpp6 [21, 26, 27]. Besides, environmental signals such as changes of carbon and nitrogen sources, $\mathrm{pH}$ can influence dimorphism of $U$. esculenta through MAPK signaling cascades $[25,28]$. Fortunately, a completely sexual cycle of $U$. esculenta has been carried out and the yeastto-mycelium dimorphic transition of $U$. esculenta can be obtained in vitro in our laboratory [25]. Based on the whole genome sequence analysis [21], it is supposed that, like $U$. maydis and other fungi [29], U. esculenta can utilize arginine as an alternative nitrogen source, through hydrolysis into ornithine and urea, which is catalyzed by arginase. In this study, we found that excess arginine inhibited the dimorphic transition of $U$. esculenta. Besides, a homologue gene coding arginase named UeArginase in $U$. esculenta was cloned and characterized. Similar to adding exogenous arginine, mutation of UeArginase could actually increase the content of endogenous arginine, and slow down the mating process, along with down regulated expressions of UePkaC, UePrf1, mfa1.2, mfa2.1, pra1 and pra2. These findings are supplements of the function of arginine on the fungal dimorphic transition, and are important for studying the interaction between $U$. esculenta and $Z$. latifolia. However, the mechanism of arginine-mediated dimorphic switching in $U$. esculenta still needs further researches.

\section{Results}

Exogenous arginine decelerates dimorphic transition

Dimorphic transition of $U$. esculenta from haploid yeast to dikaryotic filaments contains sequential phases [25]. The first typical phenotype is conjugation tubes formation, which is always regarded as the beginning of the dimorphic switching. The two heterogametic cells fused quickly through conjugation tubes and then dikaryotic filaments formed and elongated. At last, aerial hyphae extended, appeared a fuzzy appearance. Under normal mating conditions (on nutrient-rich YEPS medium), the two heterogametic strains UeT14 and UeT55 formed conjugation tubes within $12 \mathrm{~h}$, and then mated to form long hyphae with vacancies. The aerial hyphae could be seen after $24 \mathrm{~h}$, showing a white fuzzy growth (Additional file 2: Figure S1A; [25]). Hyphal length was shorter when arginine added to the YEPS medium, whereas no significant change was observed after the addition of metabolic products of arginine (urea or ornithine) (Fig. 1). Notably, higher concentration of arginine exhibited a stronger inhibition on hyphal length at 3 days after mating assays (Fig. 1). We also examined the dimorphic transition process after $10 \mathrm{mM}$ arginine added under both nutrient-rich condition (YEPS medium) and nutrient-poor condition (BM medium). When mating on BM medium, dimorphic switching began after nearly $24 \mathrm{~h}$ cultured, a $12 \mathrm{~h}$ delay compared to that on YEPS medium. After $10 \mathrm{mM}$ arginine added, a small amount of conjugation tubes formed at $24 \mathrm{~h}$ under nutrient-rich condition or at $36 \mathrm{~h}$ under nutrient-poor condition (Table 1), indicating $\mathrm{a} \sim 12 \mathrm{~h}$ delay in dimorphic switching caused by $10 \mathrm{mM}$ exogenous arginine.

\section{UeArginase is required for arginine metabolism}

Based on the whole genome shotgun sequencing (TLW00000000, 21], genes in arginine synthetic and metabolic pathway of $U$. esculenta were predicted by BlastP searching using the protein sequences of $U$. maydis [29] (Additional file 1: Table S2). At the beginning of dimorphic switching, we found that only g6606 was higher expressed in strains cultured on YESP-ARG medium, comparing to that cultured on YEPS medium (Additional file 3: Figure S2). Then, the genomic sequence and CDS sequence of $g 6606$ in $U$. esculenta were cloned (APV46198.1). It contains an open reading frame with 957 nucleotides encoding a polypeptide of 319 amino acids. Multiple sequence alignment showed that its identity to Arginase in $U$. maydis and Sporisorium reilianum is more than 90\% (Fig. 2a, Additional file 4: Figure S3). The 


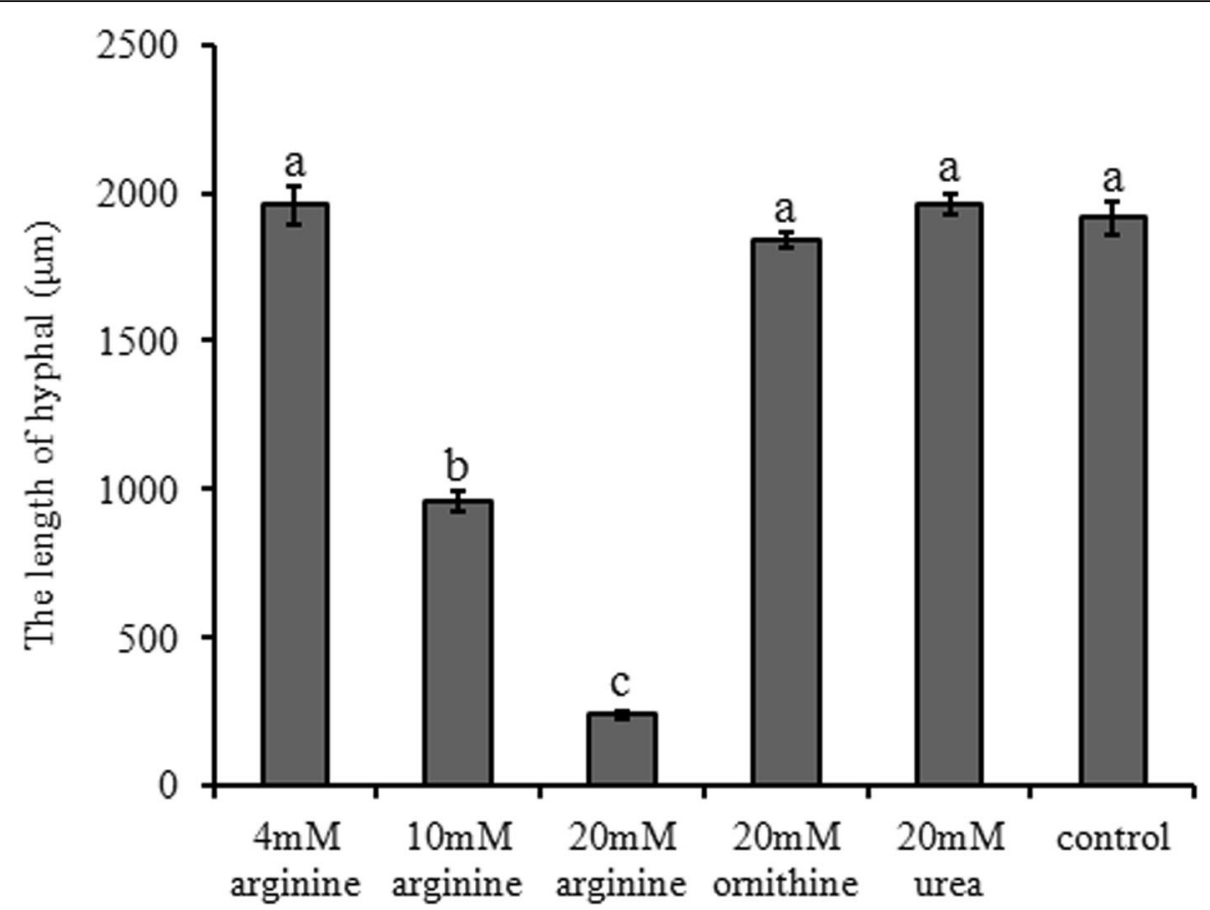

Fig. 1 The influence of arginine and its anabolites on the hyphal growth of U. esculenta. The hyphal length of the mated colonies under specified medium (X-axis) after 3 days culturing were measured under stereomicroscope. Differences in hyphal length were analyzed by One-way ANOVA. For the treatment was significant $(P<0.05)$, Tukey's multiple-comparison tests were used to analyze significant differences. Different letters above the columns indicate significant differences at $p<0.05$ level

g6606 deletion mutants were generated from WT strains (UeT14 and UeT55) using homologous recombination. Its deletion result in completely loss of arginase activity (Fig. 2b). So it is named UeArginase.

To further clarify the role of UeArginase in arginine metabolism, the changes of arginine content were tested in UeArginase-deletion mutant. According to the result of HPLC test, the content of arginine was $\sim 1.7 \mathrm{mg} / \mathrm{g}$ in WT strains, remarkably lower than that $\sim 3.5 \mathrm{mg} / \mathrm{g}$ in mutants, after $12 \mathrm{~h}$ liquid culturing in YEPS medium. When $10 \mathrm{mM}$ exogenous arginine was added to the YEPS liquid medium, the content of arginine was obviously increased both in WT strains and mutants and
UeArginase mutants. However, it reached $\sim 7.5 \mathrm{mg} / \mathrm{g}$ in mutants, $3.5 \mathrm{mg} / \mathrm{g}$ more than that in WT strains (Fig. 3). Additionally, the effect of UeArginase on the metabolism of arginine under nutrient-poor conditions was assessed., the arginine content was not significantly different between WT strains $(\sim 0.9 \mathrm{mg} / \mathrm{g})$ and UeArginase mutants $(\sim 1.3 \mathrm{mg} / \mathrm{g})$ after liquid culturing in BM mudium. When $10 \mathrm{mM}$ exogenous arginine added to BM medium, the UeArginase mutants showed an obvious defect in arginine metabolism, that the content of arginine increased by $2 \mathrm{mg} / \mathrm{g}$ in mutants, whereas that only increased by 1 $\mathrm{mg} / \mathrm{g}$ in WT strains (Fig. 3). All the data suggest that UeArginase is required for arginine metabolism.

Table 1 The conjugation formation conditions of WT strains or UeArginase mutations on YESP/YEPS-ARG/BM/BM-ARG medium after mating

\begin{tabular}{llll}
\hline Strains & Type of medium & Time of conjugation tubes formation $(\mathrm{hr})$ & Amount of the conjugation tubes \\
\hline WT & YEPS & 12 & $0.8 \pm 1.2 \%$ \\
& YEPS-ARG & 24 & $16.2 \pm 2.1 \%$ \\
& BM & 24 & $6.9 \pm 1.3 \%$ \\
& BM-ARG & 36 & $20.6 \pm 3.5 \%$ \\
VeArginase mutants & YEPS & 36 & $6.7 \pm 2.2 \%$ \\
& YEPS-ARG & 48 & $22.5 \pm 4.2 \%$ \\
& BM & 48 & $12.9 \pm 3.3 \%$
\end{tabular}



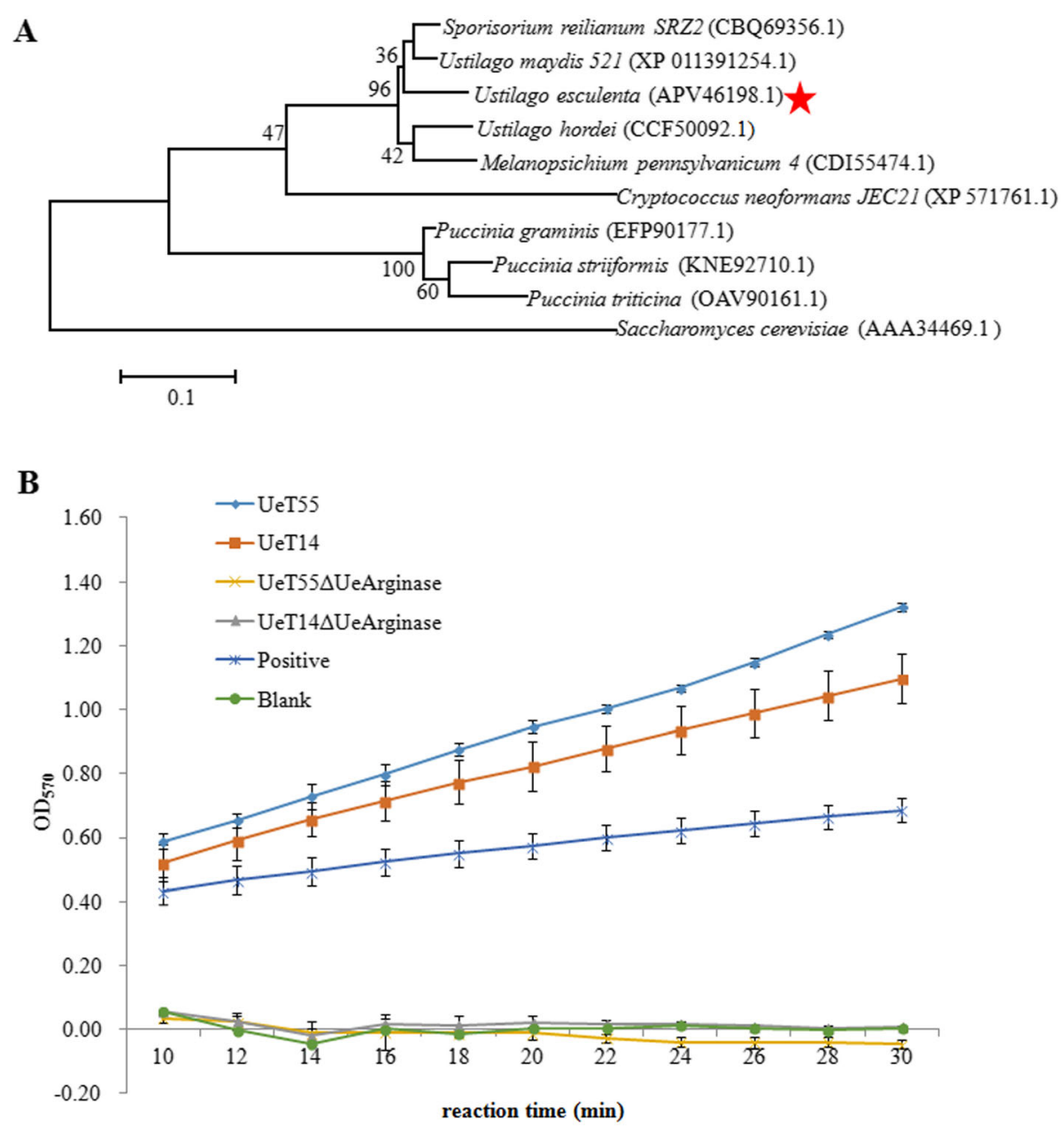

Fig. 2 Characterized of UeArginase. a Phylogenetic analysis of UeArginase. Sequence alignment was performed using ClustalW program and phylogenic tree was constructed using the neighbor-joining method. b Arginase activity was completely lost in when UeArginase was deleted. The values of $\mathrm{OD}_{570}$ in Arginase positive control increase gradually with the increase of reaction time. Greater changes of the values of $\mathrm{OD}_{570}$ in the same reaction time interval indicated a higher Arginase activity. The reaction curve of any samples similar to Blank means no Arginase activity

\section{UeArginase mutation affects the beginning of dimorphic} transition

In order to detect the role of arginine metabolism in dimorphic transition, an interval $12 \mathrm{~h}$ observation during the mating process of UeArginase mutants was carried out. Results showed that dimorphic switching of the mutants was delayed nearly $24 \mathrm{~h}$ when comparing that of the WT strains, both under nutrient-rich and nutrient-poor conditions (Table 1). In more details, during mating assays, we could see the conjugation tubes and fused cells of mutants at $36 \mathrm{~h}$ on YEPS medium and at $48 \mathrm{~h}$ on BM medium, while they appeared in WT strains at $12 \mathrm{~h}$ on YEPS medium and $24 \mathrm{~h}$ on BM medium (Additional file 2: Figure S1A, C, E, G). Similar to the WT strains, these mutants mated $12 \mathrm{~h}$ later on the medium with $10 \mathrm{mM}$ exogenous arginine than on the medium without arginine (Table 1; Additional file 2: Figure S1). Furthermore, hyphal length was measured separately at 3 days after conjugation tubes were observed. No fuzzy growth was observed when cultured on BM medium (Fig. 4a). Howerer, the length of dikaryotic filaments of either WT strains or mutants formed on BM medium was more than $4000 \mu \mathrm{m}$, nearly 2 times longer than that on YEPS medium. Besides, hyphal length of either WT strains or mutants was shorted to $\sim 700 \mu \mathrm{m}$ when mated on BMARG medium (Fig. 4b). Even more, the WT strains and mutants appeared white fuzzy growth and no obvious difference in hyphal density and length no matter cultured on YEPS medium or YEPS-ARG medium (Fig. 4b). All the data indicated that UeArginase mutation did not affect the hyphal growth.

\section{UeArginase mutation and exogenous arginine attenuates PKA-mediate signaling}

In order to explore whether the MAPK and PKA signaling pathways, proved at the heart of dimorphic transition 


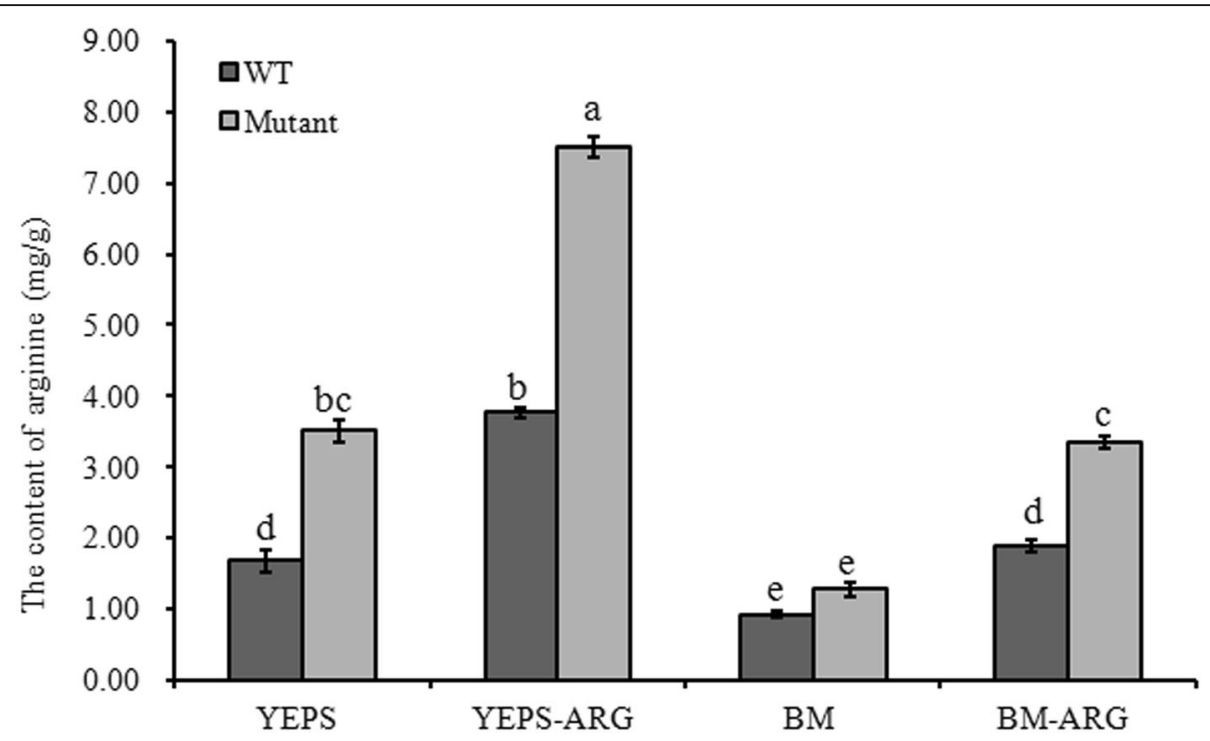

Fig. 3 The content of arginine in haploid strains of wide types or UeArginase mutations after $12 \mathrm{~h}$ liquid culturing. YEPS and BM on the X-axis represent different liquid media. -ARG represent $10 \mathrm{mM}$ arginine added to specific medium. Differences in the content of arginine were analyzed using the generalized linear model (GLM) with the variables of medium, the amount of arginine added and strains, and 3 blocks. For the interaction effect of the three variables was significant $(P<0.05)$, Least squares means were computed for multiple-comparison. Different letters above the columns indicate significant differences at $p<0.05$ (Tukey)

network [14], were affected by the UeArginase mutation during mating procedures, we further compared the expression levels of genes involved in the MAPK and PKA pathway. WT strains and mutants were tested during the mating procedure on YEPS, YEPS-ARG, BM or BM-ARG medium. UeKpp2 (KU855052) and UeKpp6 (KU855053) in MAPK pathway, UePkaC (KU302685) in PKA pathway and UePrf1 (KT343766) downstream of MAPK and PKA pathway were chosen. In the haploid strains (samples of $0 \mathrm{~h}$ in mating assays), the expression levels of UeKpp2, UeKpp6, UePkaC and UePrf1 were significant lower in mutants than those in WT strains, indicating a reduced basal expression

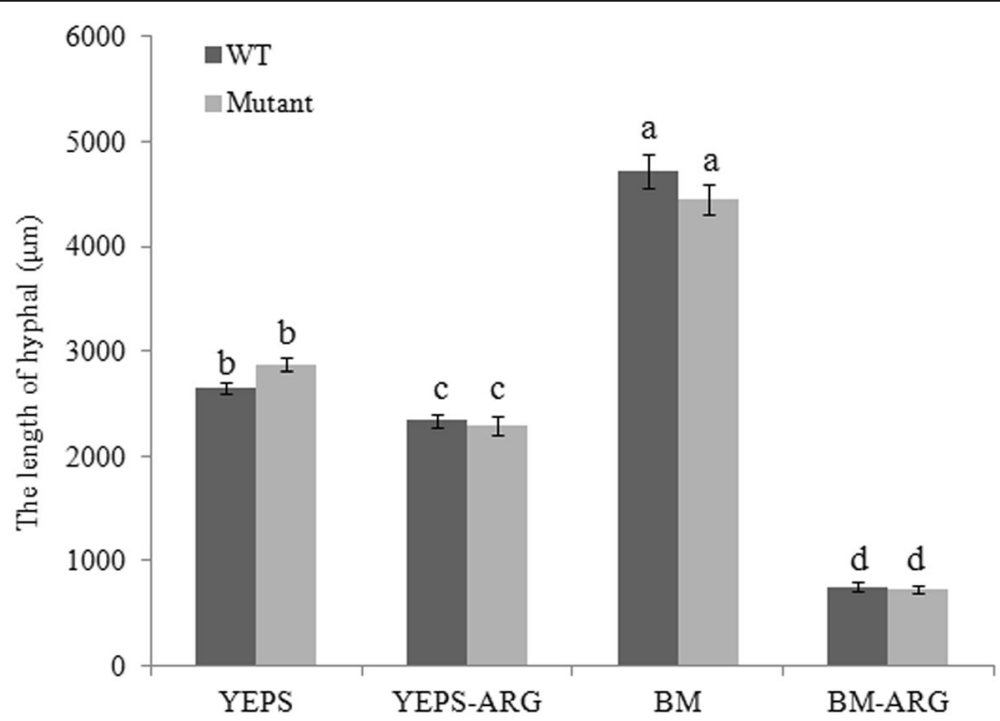

Fig. 4 The hyphal growth of WT strains or UeArginase mutations after mating. Morphology of colonies were photographed (a) and hyphal length were measured (b) under stereomicroscope at 3 days after conjugation tubes formation during mating assays carried out. Scale bar (in a) represents $1500 \mu \mathrm{m}$. YEPS and BM on the X-axis (in b) represent different liquid media. -ARG represent $10 \mathrm{mM}$ arginine added to specific medium. Differences in the hyphal length were analyzed using the generalized linear model (GLM) with the variables of medium, the amount of arginine added and strains, and 3 blocks. For the interaction effect of the three variables was significant $(P<0.05)$, Least squares means were computed for multiple-comparison. Different letters above the columns indicate significant differences at $p<0.05$ (Tukey) 
of them in UeArginase mutants (Fig. 5a-d). After $12 \mathrm{~h}$ mating on YESP medium or $24 \mathrm{~h}$ mating on BM medium (conjugation tubes were formed, indicating a dimorphic transition happened), UePrf1 and UePkaC were up-regulated both in WT strains and mutants as compared with those before mating ( $0 \mathrm{~h}$ after mating), but their expressions did not reached normal levels in mutants, less than a third of those in WT strains (Fig. 5a, b). Besides, UePrf1 and UePkaC in $10 \mathrm{mM}$ exogenous arginine treated samples were not induced after mating at $12 \mathrm{~h}$ on YESP medium or $24 \mathrm{~h}$ on BM medium (Fig. 5a, b). However, the expression levels of UeKpp 2 and UeKpp 6 were similar between WT strains and mutants after mating either in YEPS medium or $\mathrm{BM}$ medium, whether or not treated with exogenous arginine (Fig. $5 \mathrm{c}, \mathrm{d}$ ). Furthermore, the pheromone response related $a$ genes, which have been proved to be regulated by PkaC and Prf1 [30, 31], were also analyzed. The functional pheromone genes mfa1.2 (KT343772) in UeT14 and mfa2.1 (KT343776) in UeT55, and the pheromone response genes pra1 (KT343774) in UeT14 and pra2 (KT343777) in UeT55 were chosen. In the haploid strains, they showed significantly lower expression levels in mutants than those in WT strains (Fig. 5e-h). During mating, all the $a$ genes were up-regulated either in mutants or WT strains both in YEPS and BM medium, but significantly lower expression level was found in mutants than those in WT strains (Fig. 5e-h). In addition, $10 \mathrm{mM}$ exogenous arginine adding to the mating medium also inhibited $a$ genes expression after mating when compared to that mated at $12 \mathrm{~h}$ on YESP medium or $24 \mathrm{~h}$ on BM medium.

\section{Discussion}

In this study, we have elucidated the fact that arginine may function on the dimorphic transition of $U$. esculenta. In contrast to an inducer of hyphae formation in several fungi such as typical opportunistic fungal pathogen C. albicans or typical plant pathogen C. ulmi $[8,9]$, higher concentrations of arginine required for vegetative growth is negative for dimorphic transition in the endophytic-like fungus $U$. esculenta, in which the transition is nonreversible.

The inhibitory effect of arginine on dimorphic transition was first observed in $\mathrm{N}$-source bias test of $U$. esculenta [25]. As known to all, the lack of $\mathrm{N}$ sources facilitates the formation of pseudohypha or dimorphic switching in most fungi [32]. In this study, results showed that when $U$. esculenta cultured on nutritious poor medium (BM), the dimorphic switching was delayed by $12 \mathrm{~h}$ (Fig. 4a), but the hyphal length was more than 1.5 times longer, comparing to that cultured on nutritious rich medium (YESP) (Fig. 4b). According to the fact that the growth rate of $U$. esculenta in BM medium is slow [25], we thought that the lack of available $\mathrm{N}$ sources indeed promoted the elongation of mycelium, while the slowness of dimorphic transition on BM medium may be related to the cell density which is an important factor in dimorphism in many fungi [32]. When exogenous arginine added to BM or YEPS medium, the growth rate of $U$. esculenta was not influenced (Additional file 5: Figure S4), but a $12 \mathrm{~h}$ delay of dimorphic transition happened (Table 1). This was indicated exogenous arginine indeed influenced dimorphic transition. Similar findings was discovered previously that arginine and its precusor and metabolic products play important roles in fungal dimorphism $[9,14]$.

In this study, we firstly confirmed that only exogenous arginine inhibited dimorphism of $U$. esculenta, but two critical arginine anabolites ornithine and urea did not (Fig. 1, Table 1). What's more, this inhibition is proportional to the concentration of arginine (Fig. 1). Meanwhile, only UeArginase from all the arginine biosynthetic and metabolic pathway genes showed a higher expression in response to excessive exogenous arginine (Additional file 3: Figure S2). Besides, the expression of UeArginase was decreased during mating procedure (Fig. 7). Therefore, we wonder whether the arginine metabolic pathway plays the critical role in the dimorphism of $U$. esculenta. However, during mating process, UeArginase deletion mutants had no obviously different in hyphal length when compared with WT strains on the same medium (Fig. 4a). What caught our attention was that either deletion of UeArginase or exogenous arginine treatment led to increase content of arginine and delayed dimorphic transition (Fig. 4a, Table 1). Previous studies reported that excessive arginine would be stored in the vacuoles and additional arginine assimilation would induce synthetic and metabolic signals communicating between the cytosol and mitochondrial matrix and storage [33, 34]. So, we speculated that arginine itself was the influence factor and its dynamic equilibrium is critical for dimorphic transition of $U$. esculenta.

Furthermore, the content of endogenous arginine in WT strains and mutants during their mating procedure were detected by HPLC when cultured on YEPS, YEPSARG, BM or BM-ARG medium. Results showed a decrease of endogenous arginine appeared both in WT strains and mutants $12 \mathrm{~h}$ after mating on YESP or $24 \mathrm{~h}$ after mating on BM medium (Fig. 5; from $\sim 1.7 \mathrm{mg} / \mathrm{g}$ to $\sim 1.2 \mathrm{mg} / \mathrm{g}$ on YEPS medium and to $\sim 0.9 \mathrm{mg} / \mathrm{g}$ on BM medium in WT strains; from $\sim 3.5 \mathrm{mg} / \mathrm{g}$ to $\sim 2.6 \mathrm{mg} / \mathrm{g}$ on YEPS medium and to $\sim 1.3 \mathrm{mg} / \mathrm{g}$ on $\mathrm{BM}$ medium in mutants) (Fig. 6). The results indicated a decreased endogenous arginine synthesis or an increased endogenous arginine metabolism happened during dimorphic transition. Besides, the significantly reduced expression of UeArginase (Fig. 7) implicating the endogenous arginine metabolism was inhibited during mating procedure. So, a decreased endogenous synthesis may be necessary 

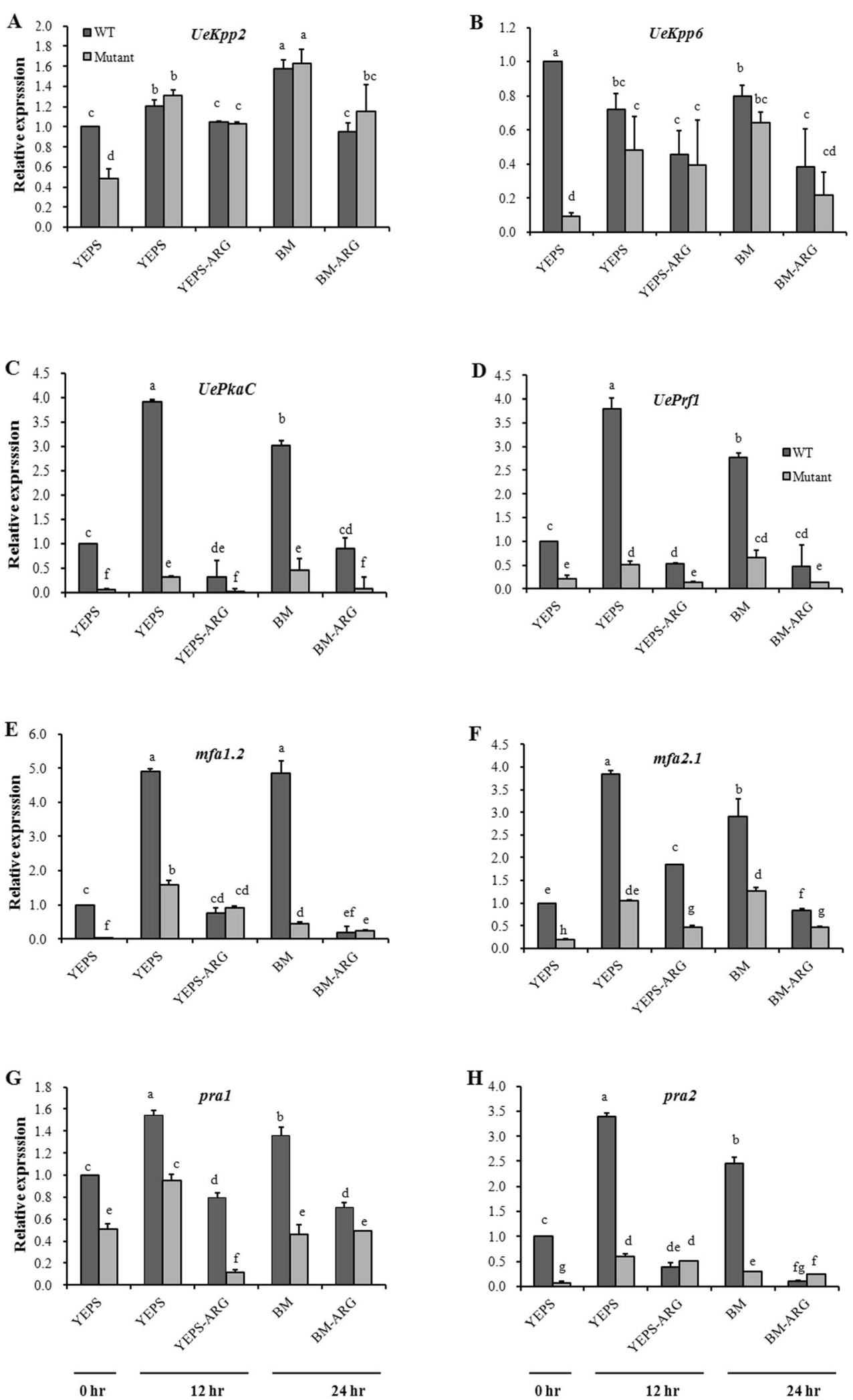

Fig. 5 (See legend on next page.) 
(See figure on previous page.)

Fig. 5 Relative expression of genes in PKA and MAPK pathway and a mating type genes during mating process. The basic expression of UeKpp2 (a), UeKpp6 (b), UePkaC (c), UePrf1 (d), mfa1.2 (e), mfa2.1 (f), pra1 (g) and pra2 (h) in WT strains and UeArginase mutations at $0 \mathrm{~h}$ and $12 \mathrm{~h}$ after mating on YESP/YEPS-ARG medium or $24 \mathrm{~h}$ after mating on BM/BM-ARG medium during mating procedure. At least 5 individual colonies were collected in the mating assay. The haploid samples mixed at the beginning of mating assays were collected as the tested samples of $0 \mathrm{~h}$. The mixed wild type strains collected at $0 \mathrm{~h}$ on YEPS medium was used as a contrast to evaluate the relative expression of genes during mating procedure. Differences in the gene expression levels were analyzed using the generalized linear model (GLM) with the variables of the stage life, medium, the amount of arginine added and strains, and 3 blocks. For the interaction effect of the four variables was significant $(P<0.05)$, Least squares means were computed for comultiple-comparison. Different letters above the columns indicate significant differences at $p<0.05$ (Tukey)

during mating. As we known, the MAPK and PKA signal pathways and $a$ genes were implicated in dimorphic transition [1, 26]. UeKpp2, UеKpp6, UePkaC, UePrf1 are key genes in these two signal pathways in $U$. esculenta $[27,28]$. Results showed a reduced basal expression of UeKpp2, UeKpp6, UePkaC, UePrf1 and a genes in mutants than those in WT strains, and a persistent lower expression levels of UePkaC, UePrf1 and $a$ genes in mutants compared to WT strains when mating on medium without arginine. Also, persistent lower expression levels of UePkaC, UePrf1 and $a$ genes appeared in strains mating on medium with arginine compared to that on medium without arginine (Fig. 5c-h).

All the data indicated that the content of endogenous arginine may be negatively correlated to the dimorphic transition and the expression of UePrf1, UePkaC and $a$ genes. These results cleared that arginine, not its metabolic products, is an inhibitor for dimorphism of $U$. esculenta, by inhibiting PKA pathway. But how arginine acts on PKA signal pathway to regulate dimorphism transition in $U$. esculenta still need much more further study.

\section{Conclusion}

In this study, we explored whether the arginine or its metabolic pathway plays the inhibition effect on the dimorphism of $U$. esculenta. Results showed that the exogenous arginine or UeArginase mutants slowed down the dimorphic transition of $U$. esculenta, along with increased content of endogenous arginine and down regulated expressions of $\mathrm{UePkaC}, \mathrm{UePrf1}$ and $a$ genes during mating process. It is speculated that arginine itself has a

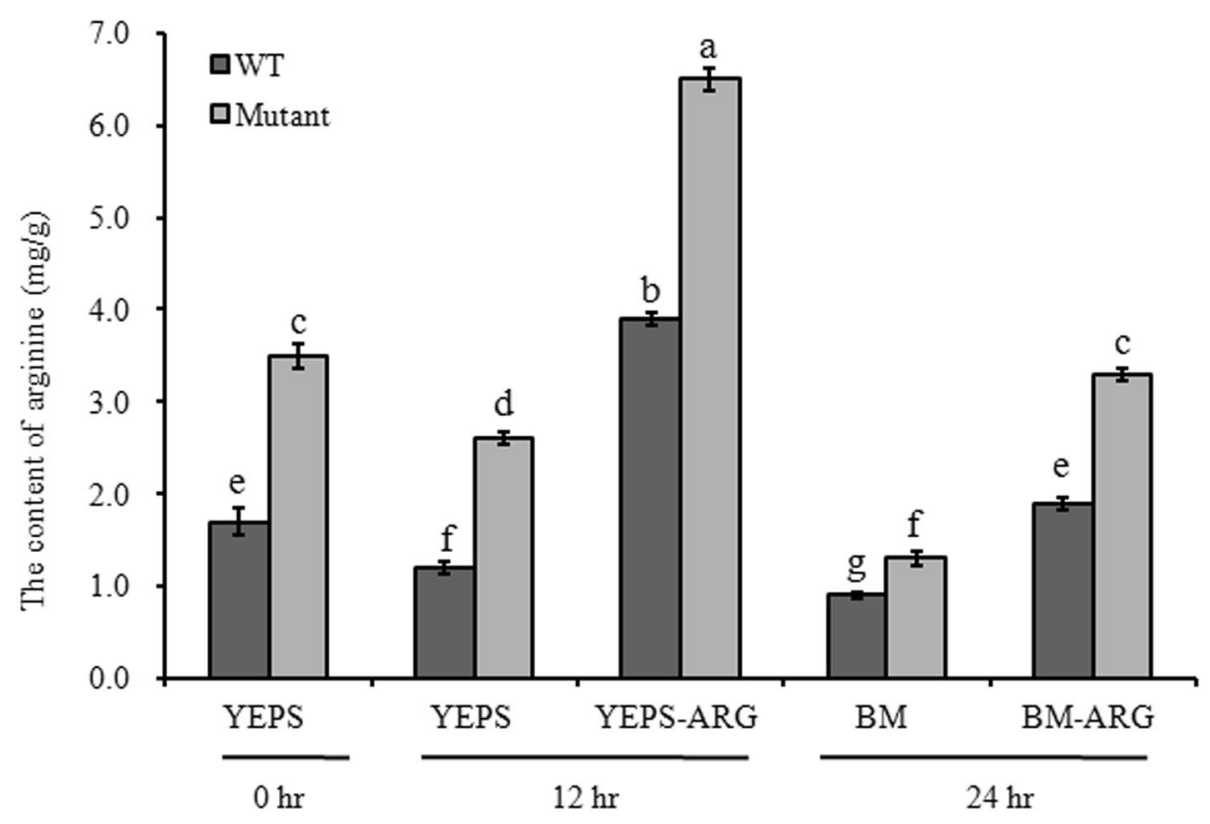

Fig. 6 The content of arginine in WT strains or UeArginase mutations during mating process. The contents of arginine in WT strains and UeArginase mutations were measured at $0 \mathrm{~h}$ and $12 \mathrm{~h}$ after mating on YESP/YEPS-ARG medium or $24 \mathrm{~h}$ after mating on BM/BM-ARG medium during mating procedure. At least 5 individual colonies were collected in the mating assay. The haploid samples mixed at the beginning of mating assays were collected as the tested samples of $0 \mathrm{~h}$. Differences in the content of arginine were analyzed using the generalized linear model (GLM) with the variables of the stage life, medium, the amount of arginine added and strains, and 3 blocks. For the interaction effect of the four variables was significant $(P<0.05)$, Least squares means were computed for comultiple-comparison. Different letters above the columns indicate significant differences at $p<0.05$ (Tukey) 


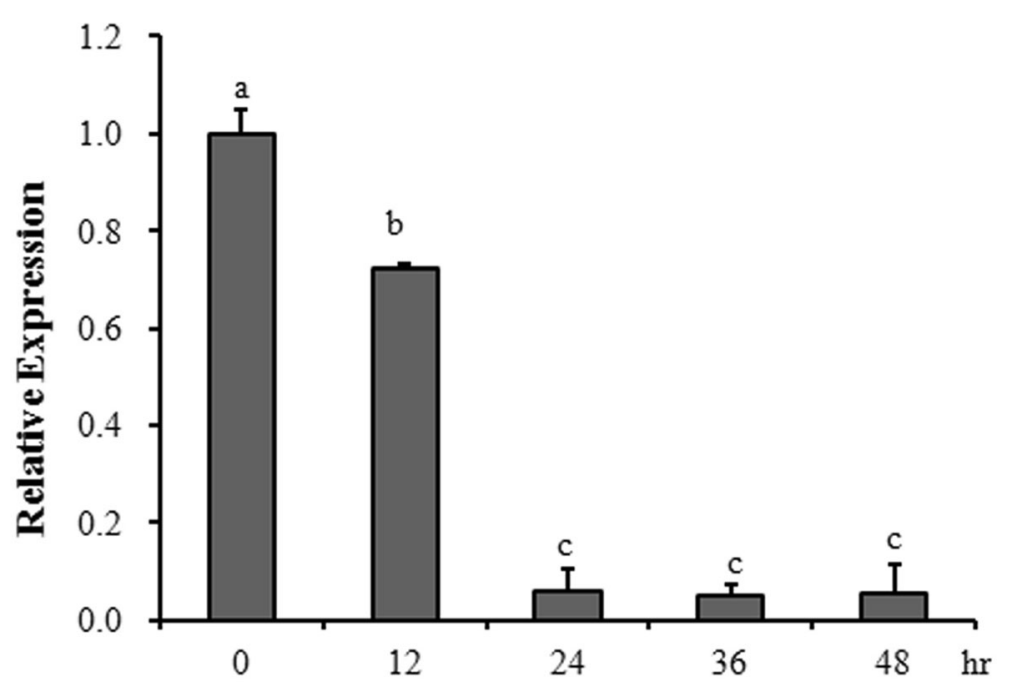

Fig. 7 Relative expression of UeArginase during mating process. At least 5 individual colonies were collected every $12 \mathrm{~h}$ in the mating assay until $48 \mathrm{~h}$. The haploid samples mixed in the beginning of mating assays were collected as the tested samples of $0 \mathrm{~h}$, which was used as a contrast to evaluate the relative expression of UeArginase during mating procedure. Differences in gene expression levels were analyzed by One-way ANOVA. For the treatment was significant $(P<0.05)$, Tukey's multiple-comparison tests were used to analyze significant differences. Different letters above the columns indicate significant differences at $p<0.05$ level

direct inhibition on the dimorphic transition of $U$. esculenta, related to its own concentration, independent of its hydrolysis by UeArginase.

\section{Methods}

\section{Strains and growth conditions}

$U$. esculenta wild type (WT) strains UeT14 (a1b1) and UeT55 (a2b2) [25] isolated from grey Jiaobai of the cultivar Longjiao 2\# (Variety number: 2,008,024 in vegetable of Zhejiang Province) in Tongxiang (30 $68^{\prime} 87.82 \mathrm{~N}$, $\left.120^{\circ} 54^{\prime} 05.49 \mathrm{E}\right)$ were used in this study. All the strains were cultured at $28^{\circ} \mathrm{C}$ on YEPS medium (yeast extract $10 \mathrm{~g} / \mathrm{L}$, peptone $20 \mathrm{~g} / \mathrm{L}$, sucrose $20 \mathrm{~g} / \mathrm{L}$ ).

\section{Gene cloning and bioinformatics analysis}

DNA was extracted using CTAB method [35]. Total RNA was extracted by Spin Column Fungal Total RNA Purification Kit (B518659, Sangon Biotech, China). cDNA was synthesized by PrimeScript ${ }^{\text {tix }}$ II 1st strand cDNA Synthesis Kit (6210A, Takara, Japan). The genomic sequence of UeArginase was identified by PCR-sequencing using the primers Arginase-gF/gR (Additional file 1: Table S1). The open reading frame (ORF) of arginase was amplified by RT-PCR with the primers Arginase-cF/cR (Additional file 1: Table S1). The intron of arginase was verified through comparing the genome sequence and ORF of UeArginase by Clone Manger program. Multiple amino sequence alignment was performed by DNAMAN with clustalW methods. Phylogenetic tree of UeArginase in $U$. esculenta and related species was constructed with the MEGA 5 programs using the neighbor-joining method.

\section{Arginase activity assay}

$1 \mathrm{~mL}$ haploid strains liquid cultured with an $\mathrm{OD}_{600}$ of 0.5 was harvest and washed by cold PBS twice. Cells were resuspended in $100 \mu \mathrm{L}$ of ice cold Assay Buffer on ice and transferred it to $1.5 \mathrm{~mL}$ tubes after grinding broken. At last, the supernatant was collected after centrifuge for $5 \mathrm{~min}$ at $4{ }^{\circ} \mathrm{C}$ at $10,000 \mathrm{x} \mathrm{g}$, and transfered to a clean tube for Arginase activity assays, according to the operation manual of Arginase Activity Assay Kit (Colorimetric, ab180877, Abcam).

\section{UeArginase deletion strains construction}

For generation of stable transformants, Hygromycin B was chosen as the selection maker and homologous recombination strategy was introduced [36, 37]. $\sim 1 \mathrm{~kb}$ fragments of the upstream and downstream of the open reading frame of UeArginase were amplified with the primers Arginase-UF1/UR1 and arginase-DF2/DR2, respectively. The hygromycin resistance gene including the promoter and terminator sequence was cloned, dividing into two fragments (one containing 5 ' sequence was amplified by paired primers Hyg-F/Hyg3-R and the other one containing 3' sequence was amplified by Hyg4-F/Hyg-R with a $25 \mathrm{bp}$ overlapping sequence). Two fragments amplified by Arginase-UF1/UR1 and Hyg-F/Hyg3-R were linked by two rounds of fusion PCR to generated the linear upstream fragment for transformation. The linear downstream fragment for transformation was generated by fusions PCR of the two fragments amplified by Hyg4F/Hyg-R and Arginase-DF2/DR2. The two constructed linear fusion fragments were used to generate UeArginase 
mutant by PEG-mediated protoplast transformation [37]. The candidate transformants would be obtained after the plate incubated at $28^{\circ} \mathrm{C}$ on regeneration medium [37] for 5-7 days and selected by normal PCR and confirmed by qRT-PCR and southern blot [27]. All the primers used were listed in Additional file 1: Table S1.

\section{Mating tests}

Colony cultured strains were expanding cultured in liquid YEPS medium with a final values of $\mathrm{OD}_{600}$ around 1.0, then collected by centrifugation and resuspended in liquid YEPS medium to an $\mathrm{OD}_{600}$ of $\sim 1.8$. Sexual compatible strains were mixed with same volume and then spotted on solid plates of test medium. Plates were sealed with parafilms and cultured at $28{ }^{\circ} \mathrm{C}$. Samples collection, observation and pictures capture under microscopy were carried out every $12 \mathrm{~h}$ over 3 days. XD Series Biological Microscope (XD30, SUNNY, China) is for observation of cell structure, such as the morphology of yeast cells, the conjugation tube and filament. SZN Zoom Stereo Microscope (EX31, SUNNY, China) is mainly used to observe the morphology of colony. Hyphal growth was observed under SZN Zoom Stereo Microscope to evaluate the mating response. Test medium prepared as follows: Basic medium (BM), including $\mathrm{K}_{2} \mathrm{HPO}_{4} 1 \mathrm{~g} / \mathrm{L}, \mathrm{MgSO}_{4} \cdot 7 \mathrm{H}_{2} \mathrm{O} 0.5 \mathrm{~g} / \mathrm{L}$, $\mathrm{FeSO}_{4} \cdot 7 \mathrm{H}_{2} \mathrm{O} 0.01 \mathrm{~g} / \mathrm{L}$ and $\mathrm{KCl} 0.5 \mathrm{~g} / \mathrm{L}$, autoclaved for sterilization, then adding $20 \mathrm{mmol} / \mathrm{L} \mathrm{KNO}_{3}$ and $50 \mathrm{mmol} /$ $\mathrm{L}$ sucrose which was filtered by Millipore filters $(0.22 \mu \mathrm{m})$; YEPS-ARG medium, including yeast extract $10 \mathrm{~g} / \mathrm{L}$, peptone $20 \mathrm{~g} / \mathrm{L}$, sucrose $20 \mathrm{~g} / \mathrm{L}$, Agar $15 \mathrm{~g} / \mathrm{L}$, autoclaved 15 min, then adding filtered L-Arginine $2 \mathrm{~g} / \mathrm{L} ; \mathrm{BM}$-ARG medium, including $\mathrm{K}_{2} \mathrm{HPO}_{4} 1 \mathrm{~g} / \mathrm{L}, \mathrm{MgSO}_{4} \cdot 7 \mathrm{H}_{2} \mathrm{O} 0.5 \mathrm{~g} / \mathrm{L}$, $\mathrm{FeSO}_{4} \cdot 7 \mathrm{H}_{2} \mathrm{O} 0.01 \mathrm{~g} / \mathrm{L}$ and $\mathrm{KCl} 0.5 \mathrm{~g} / \mathrm{L}$, autoclaved for sterilization, then adding $20 \mathrm{mmol} / \mathrm{L} \mathrm{KNO}_{3}, 50 \mathrm{mmol} / \mathrm{L}$ sucrose and $2 \mathrm{~g} / \mathrm{L}$ L-Arginine which were filtered by Millipore filters $(0.22 \mu \mathrm{m})[25]$.

\section{HPLC assay}

The content of endogenous arginine was detected by HPLC assay. Samples of mating assays were selected based on their status and hydrolyzed in hydrochloric acid for 22 $\mathrm{h}\left(0.1 \mathrm{~g}\right.$ in $1 \mathrm{~mL}$ hydrochloric acid). Then $50^{\circ} \mathrm{C} \mathrm{N}_{2}$ was used to dry all the samples. The residue dissolved in $1 \mathrm{ml}$ hydrochloric acid and filtrated by $0.22 \mu \mathrm{m}$ filter membrane. $20 \mu \mathrm{l}$ sample solution was detected under $570 \mathrm{~nm}$ and $440 \mathrm{~nm}$ channel of \#2622 $\mathrm{PH}$ columm $(4.6 \mathrm{~mm} * 60$ $\mathrm{mm}, 3 \mu \mathrm{m}$ ) in HPLC system (L-8900 Hitachi, Japan). Read the characteristic peak area at $28.667 \mathrm{~min}$. Then put it into formula $\mathrm{X}_{\mathrm{arg}}=\frac{\mathrm{C} * 174.2 * V * n * 100}{\mathrm{~m} \times 10^{6}}\left(\mathrm{X}_{\mathrm{ar}}\right.$ means the content of arginine in $100 \mathrm{~g}$ sample, $\mathrm{C}$ means the concentration of arginine which could be read through the chromatogram, $\mathrm{V}$ means the volume of hydrolysable arginine, $n$ means dilution ratio, $\mathrm{m}$ means the mass of hydrolysable arginine).

\section{Quantitative real-time PCR analysis}

Gene expression was evaluated by real-time PCR. Samples of mating assays were selected based on their status during mating on different medium. Cells were scraped from the plates to extract RNA. The PrimeScript ${ }^{\text {Tim }}$ RT reagent Kit with gDNA Eraser (Perfect Real Time, RR047A, TAKARA, Janpan) was employed for cDNA synthesis. qRT-PCR was performed on a Bio-Rad (CFX Connect ${ }^{\text {tix }}$ Real-Time System, Bio-Rad, USA) using the SYBR Premix Ex Taq ${ }^{\text {Tix }}$ (Tli RHaseH Plus, RR420A, TAKARA). The parameters and progresses were as follows: initial denaturation $95^{\circ} \mathrm{Cfor} 30 \mathrm{~s}$, followed by 40 cycles of amplification $\left(95^{\circ} \mathrm{C}\right.$ for $15 \mathrm{~s}, 58^{\circ} \mathrm{C}$ for $30 \mathrm{~s}$ ), and a melting curve at the end of each reaction consisting of a cycle $\left(95^{\circ} \mathrm{C}\right.$ for $15 \mathrm{~s}, 60^{\circ} \mathrm{C}$ for $\left.1 \mathrm{~min}\right)$ and a slow temperature increase to $95^{\circ} \mathrm{C}$ at the rate of $0.3^{\circ} \mathrm{Cs}^{-1}$. The comparative CT $\left(2^{-\triangle \Delta C T}\right)$ method was used for calculating the relative gene expression [38]. $\beta$-Actin was used as the internal reference. The primer sequences were listed in Additional file 1: Table S1.

\section{Statistical analysis}

All experiments were performed in triplicate and data were shown as mean \pm SEM from three independent experiments. All data from three independent experiments were analyzed according to the method of Student's ttest or ANOVA or generalized linear model, and $P<$ 0.05 was considered to indicate statistical significance.

\section{Additional files}

Additional file 1: Table S1. Primers used in this study. Table S2. Predicted genes in arginine synthesis and metabolic pathway. (DOCX 18 $\mathrm{kb})$

Additional file 2: Figure S1. Morphology of colonies and cells of WT strains or UeArginase mutations during mating process. The WT strains were spotted alone on YEPS (a), YEPS-ARG (b), BM (c), BM-ARG (d) plates and UeArginase mutations were spotted alone on YEPS (e), YEPS-ARG (f), BM (g), BM-ARG (h) plates. Tracing observation every $12 \mathrm{~h}$ during mating procedure was carried out until $48 \mathrm{~h}$. Typical cells morphology of indicated strains during mating was represented in the top left corner of the image of colony morphology. The scale is in the lower right corner of each image. (JPG $2360 \mathrm{~kb}$ )

Additional file 3: Figure S2. Relative expression of genes in the arginine synthesis and metabolic pathway. At least 5 individual colonies were collected at $12 \mathrm{~h}$ after mating. The samples on YEPS medium was used as a contrast to evaluate the relative expression of UeArginase during mating procedure. Differences in gene expression levels between strains cultivated in YESP/YEPS-ARG medium were analyzed by Student's t-test. Pentagonal stars above the column indicate a significant difference from others at $p<0.05$ level. (JEPG $320 \mathrm{~kb}$ ) (JPG $319 \mathrm{~kb}$ )

Additional file 4: Figure S3. Amino acid sequence alignment of UeArginase. The multiple sequence alignment was performed by DNAMAN. Blue highlighted amino acids represent 100\% identity between UePrf1 and other sequences, while green highlighted amino acids represent 75\%. (JPG $2909 \mathrm{~kb}$ )

Additional file 5: Figure S4. Comparable growth rate of UeT14 under BM or YEPS medium after exogenous arginine added. The UeT14 strain were re-suspended to an $\mathrm{OD}_{600}$ of 1.0 after liquid cultured. A cell suspension of $1 \mathrm{~mL}$ in $100 \mathrm{~mL}$ of prepared liquid medium YEPS, YEPS-ARG, 
BM, BM-ARG. Growth rates of the UeT14 were measured by OD 600 at an interval of $12 \mathrm{~h}$ culture in prepared medium. (JPG $24 \mathrm{~kb}$ )

\section{Abbreviations}

BM: Basic medium; cAMP: cyclic adenosine monophosphate; HPLC: High performance liquid chromatography; MAPK: mitogen-activated protein kinase; PKA: CAMP-dependent protein kinase A; WT: Wild type

\section{Acknowledgements}

We thank Prof. Chuanxin Sun (Swedish University of Agricultural Sciences, Sweden) for helping to modify and polish the language.

\section{Authors' contributions}

YFZ designed and guided the experiments. MW and QWG participated in the molecular studies, including genes cloning, mutation, expression et al. and the mating experiments. YFZ, MW, ZHY and QWG drafted the manuscript. MFY and SFZ performed the HPLC assay. WQX and HFC, XPY analyzed the experimental data. All authors have read and approved the final manuscript.

\section{Funding}

This work was supported by the National Natural Science Foundation of China (31600634 and 31770828). The funding bodies are play role in provide research funding of the study. They have no role in the design of the study, collection, analysis, and interpretation of data, and in writing the manuscript.

\section{Availability of data and materials}

The datasets used and analysed during the current study available from the corresponding author on reasonable request.

\section{Ethics approval and consent to participate}

Not applicable.

\section{Consent for publication}

Not applicable.

\section{Competing interests}

The authors declare that they have no competing interests.

\section{Author details}

${ }^{1}$ Zhejiang Provincial Key Laboratory of Biometrology and Inspection \& Quarantine, College of Life Sciences, China Jiliang University, Hangzhou 310018, Zhejiang, China. ${ }^{2}$ Jinhua Academy of Agricultural Sciences, Jinhua, Zhejiang, China.

Received: 10 October 2018 Accepted: 29 August 2019

\section{Published online: 05 September 2019}

\section{References}

1. Nadal M, Garcia-Pedrajas MD, Gold SE. Dimorphism in fungal plant pathogens. FEMS Microbiol Lett. 2008;284:127-34.

2. Gunasekaran S, Imbayagwo M, Mcdonald L, Gunasekaran M, Manavathu E. Influence of carbon and nitrogen sources on glutathione catabolic enzymes in Candida albicans during dimorphism. Mycopathologia. 1995;131:93-7.

3. Kulkarni RK, Nickerson KW. Nutritional control of dimorphism in Ceratocystis ulmi. Exp Mycol. 1981;5:148-54.

4. Antley PP, Hazen KC. Role of yeast cell growth temperature on Candida albicans virulence in mice. Infect Immun. 1988;56:2884-90.

5. Martínez-Espinoza AD, Ruiz-Herrera J, León-Ramírez CG, Gold SE. MAP kinase and CAMP signaling pathways modulate the $\mathrm{pH}$-induced yeast-to-mycelium dimorphic transition in the corn smut fungus Ustilago maydis. Curr Microbiol. 2004:49:274-81.

6. Munro CA, Schofield DA, Gooday GW, Gow NA. Regulation of chitin synthesis during dimorphic growth of Candida albicans. Microbiology (Reading, U K). 1998;144:391-401.

7. Blasco JL, García-Sánchez MA, Ruiz-Herrera J, Eslava AP, Iturriaga EA. A gene coding for ornithine decarboxylase (odcA) is differentially expressed during the Mucor circinelloides yeast-to-hypha transition. Res Microbiol. 2002;153:155-64.
8. Hornby JM, Jacobitz-Kizzier SM, McNeel DJ, Jensen EC, Treves DS, Nickerson KW Inoculum size effect in dimorphic fungi: extracellular control of yeast-mycelium dimorphism in Ceratocystis ulmi. Appl Environ Microbiol. 2004;70:1356-9.

9. Ghosh S, Navarathna DH, Roberts DD, Cooper JT, Atkin AL, Petro TM, et al. Arginine-induced germ tube formation in Candida albicans is essential for escape from murine macrophage line RAW 264.7. Infect Immun. 2009;77:1596-605.

10. Brefort T, Doehlemann G, Mendozamendoza A, Reissmann S, Djamei A, Kahmann R. Ustilago maydis as a pathogen. Annu Rev Phytopathol. 2009:47:423-45.

11. Froeliger $\mathrm{EH}$, Leong SA. The a mating-type alleles of Ustilago maydis are idiomorphs. Gene. 1991;100:113-22.

12. Schulz B, Banuett F, Dahl M, Schlesinger R, Schäfer W, Martin T, et al. The b alleles of $U$. maydis, whose combinations program pathogenic development, code for polypeptides containing a homeodomain-related motif. Cell. 1990;60:295-306.

13. Hartmann HA, Kahmann R, Bölker M. The pheromone response factor coordinates filamentous growth and pathogenicity in Ustilago maydis. EMBO J. 1996;15:1632-41.

14. Feldbrügge M, Kämper J, Steinberg G, Kahmann R. Regulation of mating and pathogenic development in Ustilago maydis. Curr Opin Microbiol. 2004;7:666-72.

15. Gold S, Duncan G, Barrett K, Kronstad J. cAMP regulates morphogenesis in the fungal pathogen Ustilago maydis. Genes Dev. 1994;8:2805-16.

16. Snetselaar KM, Bolker M, Kahmann R. Ustilago maydis mating hyphae orient their growth toward pheromone sources. Fungal Genet Biol. 1996;20:299-312.

17. Banuett F, Herskowitz I. Morphological transitions in the life cycle of Ustilago maydis and their genetic control by the $a$ and $b$ loci. Exp Mycol. 1994;18:247-66.

18. Hartmann HA, Krüger J, Lottspeich F, Kahmann R. Environmental signals controlling sexual development of the corn smut fungus Ustilago maydis through the transcriptional regulator Prf1. Plant Cell. 1999;11:1293-306.

19. Wösten HA, Bohlmann R, Eckerskorn C, Lottspeich F, Bölker M, Kahmann R. A novel class of small amphipathic peptides affect aerial hyphal growth and surface hydrophobicity in Ustilago maydis. EMBO J. 1996;15:4274-81.

20. Yan N, Wang XQ, Xu XF, Guo DP, Wang ZD, Zhang JZ, et al. Plant growth and photosynthetic performance of Zizania latifolia are altered by endophytic Ustilago esculenta infection. Physiol Mol Plant Pathol. 2013;83:75-83.

21. Ye ZH, Pan Y, Zhang YF, Cui HF, Jin G, McHardy AC, et al. Comparative whole-genome analysis reveals artificial selection effects on Ustilago esculenta genome. DNA Res. 2017;24:635-48.

22. Yang HC, Leu LS. Formation and histopathology of galls induced by Ustilage esculenta in Zizania latifolia. Phytopathology. 1978;68:1572-6.

23. Jose RC, Goyari S, Louis B, Waikhom SD, Handique PJ, Talukdar NC. Investigation on the biotrophic interaction of Ustilago esculenta on Zizania latifolia found in the indo-Burma biodiversity hotspot. Microb Pathog. 2016:98:6-15.

24. Piepenbring $M$, Stoll M, Oberwinkler $F$. The generic position of Ustilago maydis, Ustilago scitaminea, and Ustilago esculenta (Ustilaginales). Mycol Progress. 2002;1:71-80.

25. Zhang YF, Cao QC, Hu P, Cui HF, Yu XP, Ye ZH. Investigation on the differentiation of two Ustilago esculenta strains - implications of a relationship with the host phenotypes appearing in the fields. BMC Microbiol. 2017;17:228

26. Zhang YF, Yin YM, Hu P, Yu JJ, Xia WQ, Ge QW, et al. Mating-type loci of Ustilago esculenta are essential for mating and development. Fungal Genet Biol. 2019:125:60-70

27. Zhang YF, Liu HL, Cao QC, Ge QW, Cui HF, Yu XP, et al. Cloning and characterization of UePrf1 gene in Ustilago esculenta. FEMS Microbiol Lett. 2018;365:10

28. Zhang YF, Ge QW, Cao QC, Cui HF, Hu P, Yu XP, et al. Cloning and characterization of two MAPK genes UeKpp2 and UeKpp6 in Ustilago esculenta. Curr Microbiol. 2018;75:1016-24.

29. McCann MP, Snetselaar KM. A genome-based analysis of amino acid metabolism in the biotrophic plant pathogen Ustilago maydis. Fungal Genet Biol. 2008;45:S77-87.

30. Martinez-Soto D, Ruiz-Herrera J. Regulation of the expression of the whole genome of Ustilago maydis by a MAPK pathway. Arch Microbiol. 2015;197:575-88.

31. Choi J, Jung WH, Kronstad JW. The CAMP/protein kinase a signaling pathway in pathogenic basidiomycete fungi: connections with iron homeostasis. J Microbiol. 2015;53:579-87. 
32. Baulina O, Gorelova O, Solovchenko A, Chivkunova O, Semenova L, Selyakh I, et al. Diversity of the nitrogen starvation responses in subarctic Desmodesmus sp. (Chlorophyceae) strains isolated from symbioses with invertebrates. FEMS Microbiol Ecol. 2016;92:fiw031.

33. Guevara-Olvera L, Xoconostle-Cázares B, Ruiz-Herrera J. Cloning and disruption of the ornithine decarboxylase gene of Ustilago maydis: evidence for a role of polyamines in its dimorphic transition. Microbiology. 1997;143:2237-45.

34. Goodman I, Weiss RL. Control of arginine metabolism in Neurospora crassa. Role of feedback inhibition. J Biol Chem. 1986;261:10264-70.

35. Rogers SO. Extraction of total cellular DNA from plants, algae and fungi. In: Plant molecular biology manual D1; 1994. p. 183-90.

36. Terfrüchte M, Joehnk B, Fajardo-Somera R, Braus GH, Riquelme M, Schipper $\mathrm{K}$, et al. Establishing a versatile Golden Gate cloning system for genetic engineering in fungi. Fungal Genet Biol. 2014;62:1-10.

37. Yu JJ, Zhang YF, Cui HF, Hu P, Yu XP, Ye ZH. An efficient genetic manipulation protocol for Ustilago esculenta. FEMS Microbiol Lett. 2015;362:fnv087.

38. Livak KJ, Schmittgen TD. Analysis of relative gene expression data using real-time quantitative PCR and the 2(-Delta Delta C(T)) method. Methods. 2001;25:402-8

\section{Publisher's Note}

Springer Nature remains neutral with regard to jurisdictional claims in published maps and institutional affiliations.

Ready to submit your research? Choose BMC and benefit from:

- fast, convenient online submission

- thorough peer review by experienced researchers in your field

- rapid publication on acceptance

- support for research data, including large and complex data types

- gold Open Access which fosters wider collaboration and increased citations

- maximum visibility for your research: over $100 \mathrm{M}$ website views per year

At $\mathrm{BMC}$, research is always in progress.

Learn more biomedcentral.com/submissions 